\section{KOMPASS}

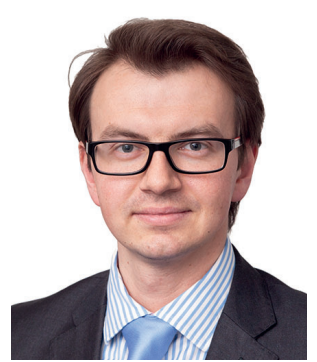

Matus Rehak

Klinik und Poliklinik für Augenheilkunde, Universitätsklinikum Leipzig AöR, Leipzig, Deutschland

Diabetes mellitus zeigt global eine rasant steigende Prävalenz. Schätzungen der Internationalen Diabetes Federation (IDF) zufolge werden im Jahr 2035 rund 592 Millionen Menschen weltweit an Diabetes erkrankt sein. Die diabetische Retinopathie (DR) ist die häufigste Komplikation von Diabetes. Etwa jeder dritte Diabetiker entwickelt im Laufe der Erkrankung eine DR, die den Annahmen zufolge von 415 Millionen Fällen im Jahr 2015 auf 642 Millionen im Jahr 2040 zunehmen wird [1]. Die DR wird anhand ihres Schweregrads in eine nicht proliferative (NPDR) und eine proliferative Form (PDR) unterschieden. Häufiger als eine PDR ist jedoch das diabetische Makulaödem (DMÖ) ursächlich für eine Visusminderung in diabetischen Patienten. Das DMÖ betrifft etwa 20 Millionen Menschen weltweit [1, 2]. Ein DMÖ kann prinzipiell bei jedem Schweregrad der DR auftreten.

In der Übersichtsarbeit dieser Ausgabe beschreiben die spanische Kollegen Olga Simó-Servat, Cristina Hernández und Rafael Simó die Risikofaktoren für die Entwicklung einer DR. Zu den bekannten Hauptrisikofaktoren gehören die Krankheitsdauer, eine schlechte Blutzuckereinstellung (hohe HbA1c-Werte) und das Vorliegen einer Hypertonie. Weiter steigt die Wahrscheinlichkeit für das Auftreten der DR bei einem hohen Body-Mass-Index (BMI), in der Pubertät oder Schwangerschaft sowie nach einer Kataraktoperation. Die Autoren weisen weiter darauf hin, dass das Vorliegen einer DR

\title{
Retinale Komplikationen von Diabetes mellitus
}

ein zuverlässiger Biomarker für die mikround makrovaskulären Komplikationen in zahlreichen Organen ist. Neue Langzeitstudien belegen, dass der kognitive Abbau bei Typ-2-Diabetikern bis zu doppelt so schnell verläuft wie die normale Alterung, und dass die Diabetiker ein erhöhtes Risiko für eine leichte kognitive Störung haben.

Auch die Rubrik «Wissenstransfer» widmet sich dem Thema der retinalen Komplikationen von Diabetes mellitus. Dr. Emiliano Di Carlo aus Karlsruhe berichtet über eine sequenzielle DMÖ-Behandlung, die aus Dexamethason-Implantat gefolgt durch Aflibercept-Injektionen besteht. Diese Vorgehensweise stellt eine alternative Behandlungsmethode zu den hochfrequenten Applikationen von Anti-VEGF-Medikamenten dar, die im klinischen Alltag oft nicht eingehalten werden können.

Die Gentherapie gilt als großer Hoffnungsträger für zukünftige Behandlungen von zahlreichen retinalen und choroidalen Dystrophien. Allerdings können die viralen Vektoren kaum die Netzhaut durchdringen, weshalb die Medikamente subretinal injiziert werden müssen. Herr PD Sebastian Siebelmann aus Köln präsentiert die Erfahrungen mit der subretinalen Applikation eines AAV-REP1-Gentherapeutikums mithilfe der Mikroskopintegrierten Optischen Kohärenztomographie (MI-OCT).

Yuhua Ding und Kollegen berichten in ihrem Fallbeispiel von einer jungen asymptomatischen chinesischen Patientin, bei der mittels multimodaler retinaler Bildgebung eine Torpedo-Makulopathie beschreiben wurde.

Ein hoch spannendes und innovatives Thema der Nutzung der künstlichen Intelligenz in der Diagnostik der retinalen Erkrankungen stellt Herr Professor Olaf Strauß aus Berlin vor. Er sieht im Einsatz der selbstlernenden Maschinen nicht nur die Möglichkeit, die Diagnostik zu verbessern, sondern die retinalen Erkrankungen besser zu verstehen und zu kontrollieren.

Der «Steckbrief Forschung» stellt die Forschung von Frau Dr. Sarah Thiele aus Bonn zum Thema «Analyse optischer Gewebeeigenschaften der Netzhaut in der SD-OCT Bildgebung im Kontext der AMD» vor. Frau Thiele ist die Trägerin des Dr.-Werner-Jackstädt Nachwuchspreises der Retinologischen Gesellschaft.

Ich wünsche Ihnen eine spannende Lektüre,

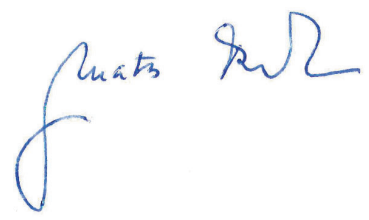

Prof. Dr. Matus Rehak, Ph.D.

\section{Literatur}

1 International Diabetes Federation: IDF Diabetes Atlas 2017, www.diabetesatlas.org (zuletzt abgerufen 17.10.2018).

2 Bandello F, Battaglia Parodi M, Lanzetta P, et al.: Diabetic Macular Edema. Dev Ophthalmol 2017;58:102-138.

\section{KARGER}

Fax +497614520714

information@karger.com

www.karger.com
(๑) 2019 S. Karger GmbH, Freiburg

Accessible online at: www.karger.com/kop
Prof. Dr. Matus Rehak

Klinik und Poliklinik für Augenheilkunde

Universitätsklinikum Leipzig AöR

Liebigstraße 10-14, 04103 Leipzig, Deutschland

matus.rehak@medizin.uni-leipzig.de 